\section{Asthma: Diagnose regelmäßig überprüfen}

Aaron SD et al. Reevaluation of Diagnosis in

Adults with Physician-Diagnosed Asthma. JAMA 2017; 317: 269 - 279

Die Sicherung einer Asthmaerkrankung kann wegen der unterschiedlichen Manifestationen und Verläufe vor allem in der hausärztlichen Praxis schwierig sein. Oft wird die (vermutete) Erkrankung empirisch behandelt, ohne dass Lungenfunktionstests erfolgen. Eine aktuelle Arbeit hat nun bei Patienten mit von einem Arzt diagnostiziertem Asthma diese Diagnose überprüft.

Shawn Aaron und seine Kollegen haben dazu zwischen Januar 2012 und Februar 2015 in den 10 größten Städten Kanadas eine Zufallsstichprobe von Patienten zusammengestellt, bei denen innerhalb der letzten 5 Jahre ein Mediziner eine Asthmadiagnose gestellt hatte. Patienten unter Behandlung mit lang wirksamen oralen Kortikosteroiden waren ausgeschlossen, ebenso starke aktuelle oder frühere Raucher, um Verzerrungseffekte durch eine mögliche chronisch obstruktive Lungenerkrankung auszuschließen.

Bei allen Studienteilnehmern führten die Wissenschaftler eine Spirometrie und Provokationstests im Hinblick auf eine bronchiale Hyperreagibilität durch. Darüber hinaus beantworteten die Patienten eine Reihe von Asthma-spezifischen Fragebogen. Bei denjenigen Patienten, die täglich Asthmamedikamente einnahmen, wurde ein schrittweises Absetzen dieser Medikamente versucht.

Als primären Endpunkt beurteilten Aaron et al. den Anteil der Patienten, bei denen ein aktuelles Asthma ausgeschlossen werden konnte. Das umfasste alle Teilnehmer, bei denen es nach Absetzen aller spezifischen Medikamente während der Nachbeobachtungszeit von 12 Monaten zu keiner Verschlechterung der subjektiven und objektiven Symptomatik kam und bei denen ein Lungenfacharzt eine alternative Diagnose gestellt hatte.
Sekundäre Endpunkte umfassten u.a. den Anteil der Patienten, bei denen bei der ursprünglichen Asthmadiagnose eine umfassende Lungenfunktionsprüfung erfolgt war.

613 der anfangs 701 Teilnehmer beendeten die Studie und konnten in die Auswertung aufgenommen werden, und bei $1 / 3$ von ihnen $(n=203)$ lag nach der obigen Definition kein Asthma vor. Im Vergleich zu Patienten mit bestätigtem Asthma hatten diese Teilnehmer

- deutlich bessere Lungenfunktionsparameter bei den Studienuntersuchungen:

- eine FEV1 (forciertes exspiratorisches Volumen) von 2,92I vor und 3,02 I nach Gabe von Bronchodilatatoren, gegenüber 2,64l bzw. $2,81 \mathrm{I}$,

- eine FEV1 von $98 \%$ des erwarteten Wertes gegenüber $88 \%$ (vor Bronchodilatatoren) und von $101 \%$ gegenüber $93 \%$ (nach Bronchodilatatoren) sowie

- keine relevante Zunahme der FEV1 nach Gabe von Bronchodilatatoren, definiert als Zahl der Patienten mit Zunahme $\geq 12 \%$ und $\geq 200 \mathrm{ml}$

(0 gegenüber 86 Patienten)

- seltener insgesamt und seltener täglich Asthmamedikamente eingenommen $(79,3 \%$ vs. $90,2 \%$ und $35,0 \%$ vs. $49,3 \%)$ und

- bei der ersten Diagnose seltener eine Spirometrie erhalten (43,8\% vs. $55,6 \%)$.

In der multivariaten logistischen Regressionsanalyse waren nach Adjustierung im Hinblick auf Alter, Geschlecht und BodyMass-Index

- eine geringer geschätzte FEV1 (Odds Ratio [OR] 0,95 pro Zunahme um 1\%),

- die tägliche Einnahme von Asthmamedikamenten (OR 1,63),

- die objektive Sicherung der Exspirationsbehinderung bei der ursprünglichen Diagnose (OR 1,79) und

- anamnestisch in den letzten 12 Monaten aufgetretene pfeifende Atmung (OR 2,57)

mit einer erhöhten Wahrscheinlichkeit für eine Bestätigung der Asthmadiagnose verbunden.

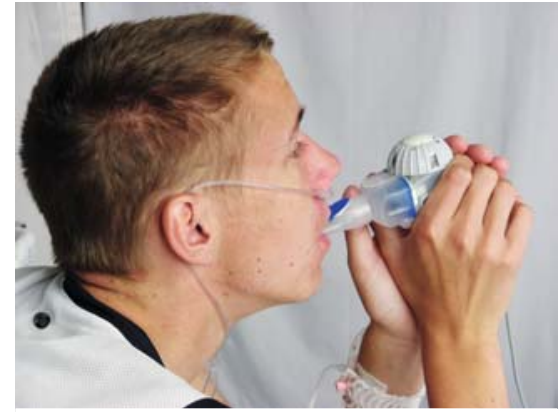

Laut vorangehender Studien wurde bei weniger als der Hälfte der Patienten, die mit Asthma diagnostiziert wurden, eine Spirometrie zur Feststellung einer variablen exspiratorischen Atemwegsobstruktion durchgeführt. Bildnachweis:

M. Wettstein/Thieme Verlagsgruppe.

Die statt des Asthmas von den Lungenfachärzten gestellten alternativen Diagnosen umfassten meist unkomplizierte Störungen, aber bei immerhin 12 Patienten wurden schwere kardiorespiratorische Erkrankungen diagnostiziert. Darunter waren 4 Patienten mit koronarer Herzkrankheit, 2 mit subglottischer Stenose, 2 mit Bronchiektasen und jeweils 1 mit interstitieller Lungenerkrankung, pulmonalarterieller Hypertonie, Sarkoidose und Tracheobronchomalazie.

FAZIT

Etwa $1 / 3$ der Erwachsenen, bei denen ambulant eine Asthmadiagnose gestellt wurde, zeigte bei der Kontrolle keine Erkrankungssymptome, so die Autoren. Daher sollte nach 3 Monaten guter Symptomkontrolle versucht werden, die Medikamente auszuschleichen - so wie es auch die Leitlinien der Fachgesellschaften vorsehen. Zusätzlich sei eine objektive Sicherung der Diagnose mittels Spirometrie zu empfehlen. Das könnte Patienten unnötige Nebenwirkungen und dem Gesundheitssystem unnötige Kosten ersparen.

Dr. Elke Ruchalla, Bad Dürrheim 